\title{
Signal enhancement techniques for if SQUID based magnetic imaging systems
}

To cite this article: Rizwan Akram et al 2006 Supercond. Sci. Technol. 19821

View the article online for updates and enhancements.

Related content

Front-end Assembly Optimization for HighTrrf-SQUID based Magnetic Field Imaging Systems

R Akram, M Fardmanesh, J Schubert et al.

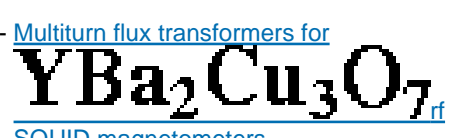

SQUID magnetometers

H R Yi, J Schubert, W Zander et al.

- The stability of dc and if SQUIDs in static ambient fields

V N Glyantsev, Y Tavrin, W Zander et al.

\section{Recent citations}

\begin{tabular}{l} 
- Effects of Temperature, Thickness and \\
Bias Current on Magnetoelectric \\
\hline Characteristics of Silicon Micro-Hall \\
\hline Sensors \\
Rizwan Akram \\
- Low noise SQUID based NDE with non- \\
magnetic Scanning system in unshielded \\
environment \\
Y Khatami et al
\end{tabular}

\section{IOP ebooks}

Bringing you innovative digital publishing with leading voices

to create your essential collection of books in STEM research.

Start exploring the collection - download the first chapter of every title for free. 


\title{
Signal enhancement techniques for rf SQUID based magnetic imaging systems
}

\author{
Rizwan Akram ${ }^{1,6}$, Mehdi Fardmanesh ${ }^{2,3}$, Juergen Schubert ${ }^{4}$, \\ Willi Zander ${ }^{4}$, Marko Banzet ${ }^{4}$, Dieter Lomparski ${ }^{4}$, \\ Miak Schmidt ${ }^{4,5}$ and H-J Krause ${ }^{4}$ \\ ${ }^{1}$ Department of Physics, Izmir Institute of Technology, Urla Tr-35430, Izmir, Turkey \\ ${ }^{2}$ Electronics Engineering Department, Bilkent University, Ankara 06533, Turkey \\ ${ }^{3}$ Electrical Engineering Department, Sharif University of Technology, Tehran, Iran \\ ${ }^{4}$ ISG1-IT, Forschungszentrum Juelich GmbH, D-52425 Juelich, Germany \\ ${ }^{5}$ Biotechnological Biomedical Centre, University of Leipzig, Deutscher Platz 5, 04103 \\ Leipzig, Germany \\ E-mail: rizwanakram75@gmail.com
}

Received 4 April 2006, in final form 11 June 2006

Published 3 July 2006

Online at stacks.iop.org/SUST/19/821

\begin{abstract}
We have investigated the rf SQUID (radio-frequency superconducting quantum interference device) and its coupling to tank circuit configurations to achieve an optimal front-end assembly for sensitive and high spatial resolution magnetic imaging systems. The investigation of the YBCO rf SQUID coupling to the conventional $L C$ tank circuits revealed that coupling from the back of the SQUID substrate enhances the SQUID signal while facilitating the front-end assembly configuration. The optimal thickness of the substrate material between the SQUID and the tank circuit is $0.4 \mathrm{~mm}$ for $\mathrm{LaAlO}_{3}$ resulting in an increase of the SQUID flux-voltage transfer function signal, $V_{\text {spp }}$, of 1.5 times, and $0.5 \mathrm{~mm}$ for $\mathrm{SrTiO}_{3}$ with an increase of $V_{\text {spp }}$ of 1.62 times compared to that for direct face to face couplings. For rf coupling with a coplanar resonator, it has been found that the best configuration, in which a resonator is sandwiched between the SQUID substrate and the resonator substrate, provides a $V_{\mathrm{spp}}$ about 3.4 times higher than that for the worse case where the resonator and the SQUID are coupled back to back. The use of a resonator leads to a limitation of the achievable spatial resolution due to its flux focusing characteristics. This resulted in a favouring of the use of the conventional tank circuits when considering the desired high spatial resolution. The effect of the YBCO flip chip magnetic shielding of the SQUIDs in the back-coupling with the $L C$ tank circuit configuration has also been investigated, with a view to reducing the SQUID effective area to increase the spatial resolution and also for studying the effect of the coupling of various kinds of transformers to the SQUIDs. It is revealed that there is no very considerable change in the flux-voltage transfer function signal level with respect to the effective shield area, while the lowest working temperature of the SQUIDs was slightly shifted higher by a couple of degrees, depending on the shield area.
\end{abstract}

(Some figures in this article are in colour only in the electronic version)

\section{Introduction}

For sensitive applications of rf SQUID based magnetic imaging systems, where high magnetic field sensitivity with high spatial

6 Author to whom any correspondence should be addressed. resolution is required, two important issues to be considered are the front-end assembly of the rf SQUID based system and the rf SQUID flux to voltage transfer function signal level, $V_{\text {spp }}[1,2]$. With respect to the system design, use of high $T_{\mathrm{c}}$ SQUIDs is superior compared to that of low $T_{\mathrm{c}}$ devices, 
mainly due to the lift-off distance (LOD), the distance between the SQUID and the sample, which plays a vital role for frontend assembly. LOD is inevitably widened for helium based systems, whereas it can be decreased to a few tens of microns in nitrogen based systems by using a thin window. Designing such a system offers serious challenges especially when it is required to work for samples at room temperature, which furthermore requires a precise separation adjustment system. Bottlenecks allied to such applications of the rf SQUID based systems are highlighted and investigated in this work. In this study we have probed some of these limiting factors, which not only provides insight for improving the sensitivity and field resolution, but also helps in the development of new configurations for the front-end assembly of high resolution magnetic field imaging systems [3-5].

\section{Sample preparation and the experimental set-up}

The rf SQUID magnetometers and gradiometers were made using YBaCuO film deposited on $\mathrm{LaAlO}_{3}(100)$ and $\mathrm{SrTiO}_{3}$ (100) substrates. The $\mathrm{YBaCuO}$ films were deposited by pulsed laser deposition techniques using a $\mathrm{KrF}$ excimer laser and were patterned using either wet chemical etching in $0.25 \%$ dilute phosphoric acid or a low energy argon IBE process. The rf washer SQUIDs were made either using step edge junctions or bicrystal grain boundary junctions. The magnetometers were made using a layout with a $3.5 \mathrm{~mm}$ diameter washer and a loop size of $150 \times 150 \mu \mathrm{m}^{2}$. The gradiometers were made using layouts with washer areas with $1.5 \mathrm{~mm}$ diameter, loop areas of $75 \times 75 \mathrm{~cm}^{2}$ and a baseline of $1.5 \mathrm{~mm}$ [6].

During our study, a coplanar resonator and flux concentrator was fabricated on a standard STO substrate coated with a $200 \mathrm{~nm}$ layer of YBCO film. An rf SQUID is placed on the $10 \times 10 \times 1 \mathrm{~mm}^{3}$ substrate to form a flip chip package [7]

\section{Analytical coupling techniques and improving techniques}

\subsection{Coupling techniques advantages and disadvantages}

Both field sensitivity and spatial resolution for SQUID microscopes are highly dependent on the distance between the sensor and the sample. Hence, we have investigated various approaches for coupling the rf SQUID sensor and the samples to find the optimal configuration. The high field sensitivity of high $T_{\mathrm{c}}$ rf SQUIDs is associated with their large area layout; this area inherently hinders achieving high spatial resolution. The washer area of the rf SQUIDs must be optimized for the minimum area needed for efficient coupling to the rf coupling circuit [5].

As a replacement for the conventional $L C$ tank circuit, which not only provides less $V_{\text {spp }}$ but also takes more space, thus hindering the front-end assembly, thin film superconducting coplanar resonators were used and investigated in the flip chip configuration $[7,8]$. In the case of the coplanar resonator, we used either an $8 \mathrm{~mm}$ rectangular (SR8) or a $13.4 \mathrm{~mm}$ circular (SR13) layout design with integrated flux concentrators [7]. The difference between the performances of the rf SQUIDs using these couplings was investigated. As shown in figure 1 , the $V_{\mathrm{spp}}$ is an order of

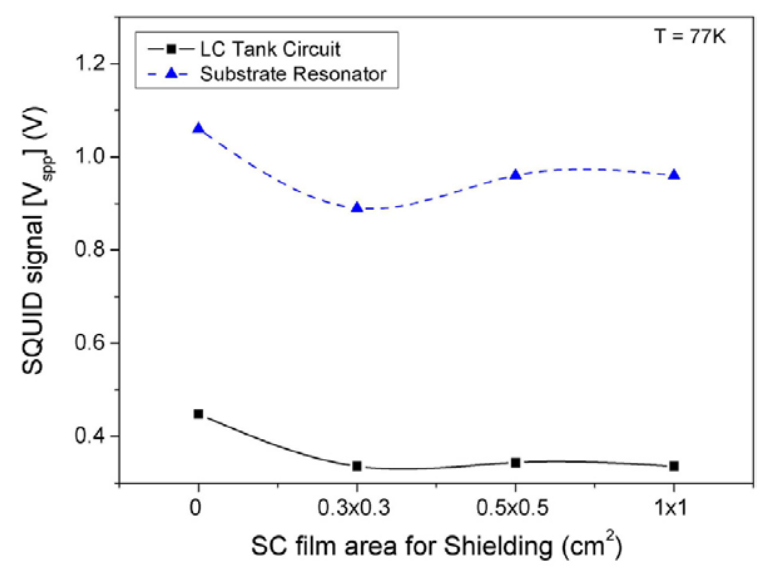

Figure 1. Comparison between the tank circuit and resonator for the rf SQUID signal level under different shielding conditions. A shielding SC film has been applied in the flip chip configuration.

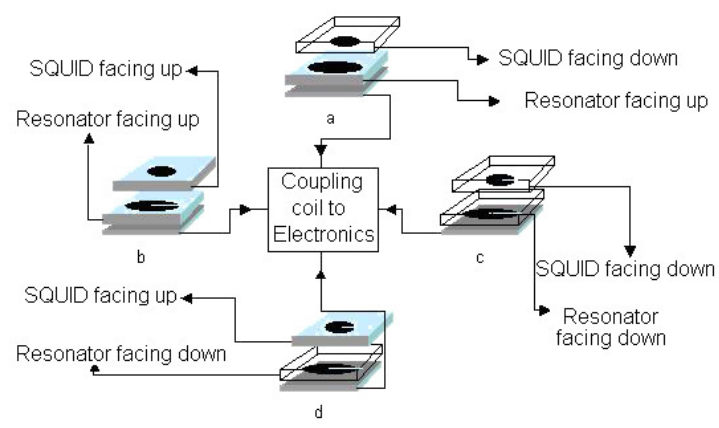

Figure 2. Basic possible configurations for the resonator to rf SQUID coupling.

magnitude higher in the case of coplanar resonator compared to a $L C$ tank circuit. The noise level of the rf SQUIDs has been found to be almost independent of the type of $\mathrm{rf}$ coupling technique [9], favouring the use of the tank circuit when considering the desired high spatial resolution. It is to be noted that the use of coplanar resonators with integrated flux concentrators introduces serious limitations in the spatial resolution. The possibilities for coupling with the coplanar resonator that were investigated are shown in figure 2.

\subsection{Signal improvement techniques}

Results obtained from our systematic study of the techniques of coupling for the resonator, rf SQUID and pick-up coil of the electronics are listed in table 1. In the type ' $\mathrm{B}$ ' configuration of figure 2 , where there is a substrate between the rf SQUID and the resonator and a substrate between the resonator and the pick-up coil, it has been observed that both higher SQUID signal level and noise reduction can be achieved. The improvement in the signal level compared to the worse case, D, is about 3.4 times. These results provide insight into two important facts:

(a) a distance between the resonator and the coupling coil provides better signal coupling with the electronics;

(b) dielectric material between the SQUID and the resonator improves the rf SQUID signal level, $V_{\text {spp }}$. 
Signal enhancement techniques for rf SQUID based magnetic imaging systems

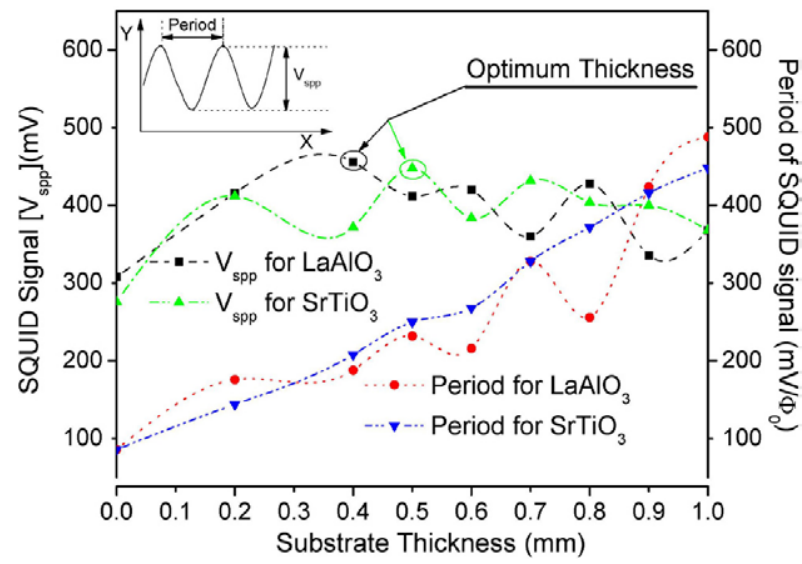

Figure 3. Effect of substrate $\left(\mathrm{LaAlO}_{3}\right.$ and $\left.\mathrm{SrTiO}_{3}\right)$ thickness between rf SQUID and $L C$ tank circuit. The inset explains the terms period and $V_{\mathrm{spp}}$, from the $x-y$ graph where the $x$-axis represents the input applied modulation flux and the $y$-axis represents the output of the rf SQUID electronics.

Table 1. Possible rf SQUID and the coplanar resonator coupling configurations with their relative signal and signal locking states (S: SQUID, R: resonator, sub: substrate, C: pick-up coil).

\begin{tabular}{llll}
\hline Type & Description & $V_{\text {spp }}$ level & Quality \\
\hline A & S-R-sub-C & $900 \mathrm{mV}$ & Lockable \\
B & S-sub-R-sub-C & $1.18 \mathrm{~V}$ & Lockable and less noisy \\
C & S-sub-R-C & $400 \mathrm{mV}$ & Lockable and noisy \\
D & S-sub-sub-R-C & $350 \mathrm{mV}$ & Barely lockable and noisy \\
\hline
\end{tabular}

\subsection{The effect of the substrate thickness on the rf SQUID signal}

As theoretically expected [10] and seen in table 1, the SQUID signal improves on introducing dielectric material in the gap between the coupling circuit and the rf SQUID for both cases of coplanar resonators and the $L C$ tank circuit. This effect has been investigated by introducing substrate materials with various thicknesses, between the rf SQUID and the $L C$ tank circuit, for the two kinds of substrate materials used, namely $\mathrm{LaAlO}_{3}$ and $\mathrm{SrTiO}_{3}$. The effect of the substrate thickness on the SQUID signal, $V_{\text {spp }}$, for both $\mathrm{LaAlO}_{3}$ and $\mathrm{SrTiO}_{3}$ materials is shown in figure 3. As shown in the figure, the optimum substrate thickness was found to be about $0.4 \mathrm{~mm}$ for $\mathrm{LaAlO}_{3}$ and $0.5 \mathrm{~mm}$ for $\mathrm{SrTiO}_{3}$ substrate materials. To reach the optimal substrate thicknesses one should thin the substrates either prior to the SQUID fabrication or after. Both techniques gave satisfactory results $[11,12]$. Devices with various substrate thicknesses were made, and characterized and presented elsewhere [12].

\section{Shielding characteristics}

\subsection{The effect of shielding and the temperature effect}

It has been observed that noise coupling from the resonator circuit is very influential in most of the applications. This is especially the case when pick-up transformers are being used to increase the spatial resolution [11]. In such cases, field sensing is not only due to the SQUID but also due to the resonator and the transformer legs which serve as sensing

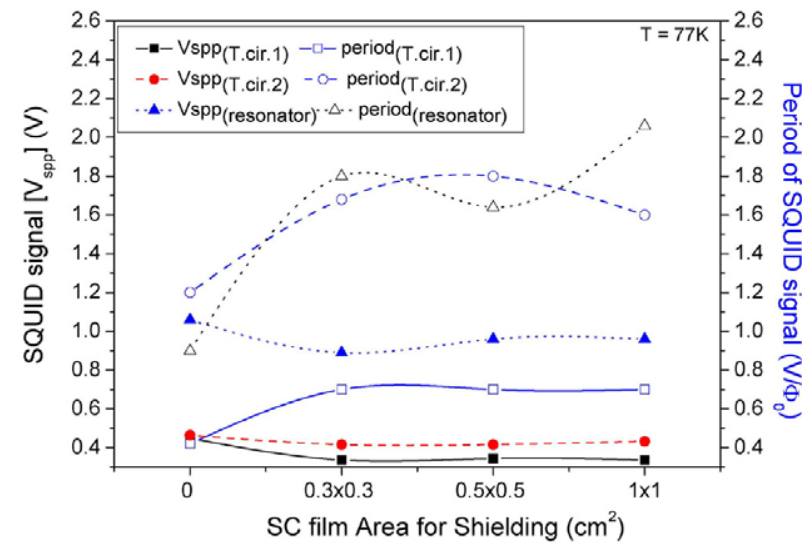

Figure 4. Effect of the applied shield area (a). Tank circuits 1 and 2 are conventional $L C$ tank circuits but the loop diameter of the inductor for tank circuit 1 is $\sim 0.5 \mathrm{~mm}$ and for 2 it is $\sim 1 \mathrm{~mm}$. The resonator circuit of the conventional superconducting circular washer type.

devices, and add to the noise. To overcome this hurdle, the solution of shielding the unwanted areas has been investigated in detail. In order to determine the shielding factor, rf SQUIDs were covered with a $1 \mathrm{~cm}^{2} \mathrm{YBaCuO}$ thick film acting as a magnetic shield. The theoretical expectation for the shielding factor of a $1 \times 1 \mathrm{~cm}^{2}$ shield applied to a rf SQUID through a substrate of about $1 \mathrm{~mm}$ thickness is about 10 [10], whereas from experimentally obtained data show a best result of 8.67 for a small washer area rf SQUID [4, 11]. The measured shielding factor shows an inverse dependence on the SQUID's washer area and it shows linear behaviour with respect to the applied field. This leads to the observation that proper shielding of the SQUID can be achieved by having a smaller washer area for the case when a transformer is used to pick the signal up. The possibility of suppressing the SQUID signal by applying a shield in different coupling techniques has been investigated by covering the rf SQUID washer area with $200 \mathrm{~nm}$ thick YBaCuO film. The measured unlocked signal of the test SQUID is shown in figure 4.

From figure 4, for tank circuit 1, it is clear that an increase of the shield area from 0 to $1 \mathrm{~cm}^{2}$ causes about a $16.2 \%$ decrease in $V_{\text {spp}}$, while the period (inset of figure 3 ) of the SQUID signal is increased by $82 \%$. Therefore, for applications, where $V_{\text {spp }}$ is being used for measurement, shielding does not very considerably affect the overall measurement. It has also been observed that application of the rf coupling technique not only affects the SQUID signal level, but also causes suppression in the signal level when applying a shield. It is clear from figure 4 that a coplanar resonator provides a 2.28 times better signal level compared to that obtained by the $L C$ tank circuit. But on the other hand, shielding adversely affects the resonator coupling by decreasing the signal level by $10-20 \%$, whereas in an optimized $L C$ tank circuit, it is just about $7 \%$, depending on the applied shield area.

\subsection{The effect of the shielding on different coupling techniques}

In order to investigate the reason for the reduction in the signal of the SQUID produced by the use of the shield, the 


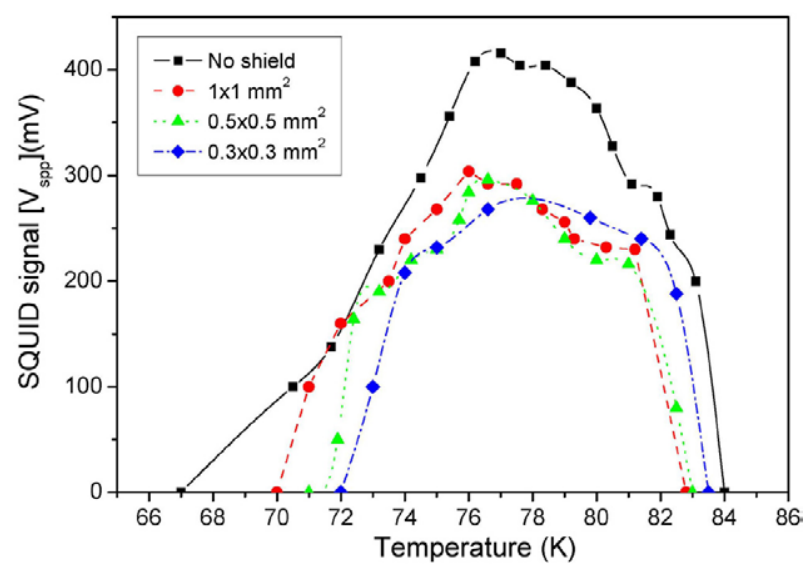

Figure 5. Effect of the temperature on the $V_{\text {spp }}$ of the shielded rf SQUID.

temperature sensitivity versus shielding effect on $V_{\mathrm{spp}}$ for the devices has been measured and is shown in figure 5 . Theoretically, the inductance of the SQUID is changed by applying a shield, and this inductance change of the SQUID loop causes a change in the bias point. In such a situation, by increasing the temperature we return the SQUID signal back to same value, which relates to the need of a SQUID with higher $J_{\mathrm{c}}$ than that calculated for an unshielded SQUID. In this way the inductance and current product will remain the same under shielding for the optimum temperature, which in our case is $77 \mathrm{~K}$. From figure 5, it is clear that such an intersection point can be found by increasing the temperature, where the signal with the shield is equal to that without the shielding. This leads to the need for further optimization of an rf SQUID design with the desired $J_{\mathrm{c}}$ for optimum operation under shielding conditions, which is under investigation.

\section{Summary and conclusions}

The bottlenecks related to the applications of the rf SQUID for high spatial and high field sensitivity resolutions have been systematically investigated. In this study, liquid nitrogen based systems were used since they provide a better solution than liquid helium based systems due to the possibility of just using the thin window concept to reduce the lift-off distance. Both the coplanar resonator and the conventional $L C$ tank circuit based configurations were investigated. Use of coplanar resonators has been found to provide better signal levels compared to conventional $L C$ tank circuits for bare rf SQUIDs without an YBCO film shield. However for the usage under shielding, a conventional $L C$ tank circuit with appropriate optimization is advantageous, based on lower suppression of the rf SQUID signal and lower unwanted pick-up which cannot be avoided in the case of a coplanar resonator due to its large flux focusing area. The $L C$ tank circuit also provides a lower percentage suppression under shielding compared to the coplanar resonators. The shielding effect on coupling techniques and with respect to temperature on the $V_{\text {spp }}$ has also been investigated. This investigation can be concluded as follows:

(a) according to our speculation, film used for shielding should be as thick as possible $(\sim 400-500 \mathrm{~nm})$, so that a better shielding factor can be achieved,

(b) SQUIDs with relatively high $J_{\mathrm{c}}$ are required, as under shielding the reduction in the inductance caused by the shield is compensated.

\section{References}

[1] Enpuku K, Minotani T, Hotta M and Nakahodo A 2001 Application of high $T_{\mathrm{c}}$ SQUID magnetometer biological immunoassays IEEE Trans. Appl. Supercond. 11 661-4

[2] Lee T S, Chemla Y R, Dantsker E and Clarke J 1997 High $T_{\mathrm{c}}$ SQUID microscope for room temperature samples IEEE Trans. Appl. Supercond. 7 3147-50

[3] Bousack H, A SQUID 9-channel module for application in biomagnetics, nondestructive evaluation and geomagnetism, ISI, Research Centre Juelich GmbH, Germany, Final report 13N7327iA (in German)

[4] Akram R 2005 Fabrication and optimization of rf-SQUID and Integration assembly for high resolution magnetic imaging system PhD Thesis Bilkent University, Ankara, Turkey

[5] Schmidt M, Krause H-J, Banzet M, Lomparski D, Schubert J Zander W, Zhang Y, Akram R and Fardmanesh M 2006 The set-up of an high temperature superconductor radio-frequency SQUID microscope for magnetic nanoparticle detection Supercond. Sci. Technol. 19 s261-5

[6] Fardmanesh M, Schubert J, Akram R, Banzet M, Zander W, Zhang Y, Schilling M and Krause H-J 2002 Dependence of the substrate structure and the film growth at the junction of YBCO SEJ rf-SQUIDs on the IBE process and effects on the SQUID's characteristics Physica C 372 240-4

[7] Zhang Y, Wolters N, Zeng X H, Schubert J, Zander W, Soltner H, Zi H R, Banzet M, Ruder F and Braginski A I 1998 Washer rf-SQUID magnetometers with coplanar resonators at 77k Appl. Supercond. $6385-90$

[8] Zhang Y, Zander W, Schuber J, Ruders F, Soltner H, Banzet M, Wolters N, Zeng X and Braginski A I 1997 Operation of high sensitivity rf SQUID magnetometers with superconducting coplanar resonators at 77k Appl. Phys. Lett. 77 704-6

[9] Fardmanesh M, Schubert J, Akram R, Bick M, Zhang Y, Banzet M, Zander W, Krause H-J, Burkhart H and Schilling M 2001 1/f noise characteristics of SEJ $\mathrm{Y}-\mathrm{Ba}-\mathrm{Cu}-\mathrm{O}$ rf-SQUIDs on $\mathrm{LaAlO}_{3}$ substrate and the step structure, film, and temperature dependence IEEE Trans. Appl. Supercond. 11 1363-6

[10] Duzer T V and Turner C W 1999 Principles of Superconductive Devices and Circuits 2nd edn (Englewood Cliffs, NJ: Prentice-Hall)

[11] Akram R 2003 Development and optimization of HTC SQUID technology for a high resolution magnetic imaging, Juelich (FZJ-ISG2) and Bilkent (EEE dept.), Joint Research and development project, 2003

[12] Schmidt M 2003 SQUID mikroskop zur detektion magnetischer nanopartikel Diplomarbeit Forschungszentrum Juelich 